\title{
Neutron irradiation of coated conductors
}

\author{
Eisterer M, ${ }^{1, *}$ Fuger R, ${ }^{1}$ Chudy M, ${ }^{1}$ Hengstberger F, ${ }^{1}$ and Weber $\mathrm{H} \mathrm{W}^{1}$ \\ ${ }^{1}$ Atominstitut, Vienna University of Technology, Stadionallee 2, 1020 Vienna
}

\begin{abstract}
Various commercial coated conductors were irradiated with fast neutrons in order to introduce randomly distributed, uncorrelated defects which increase the critical current density, $J_{\mathrm{c}}$, in a wide temperature and field range. The $J_{\mathrm{c}}$-anisotropy is significantly reduced and the angular dependence of $J_{\mathrm{c}}$ does not obey the anisotropic scaling approach. These defects enhance the irreversibility line in not fully optimized tapes, but they do not in state-of-the-art conductors. Neutron irradiation provides a clear distinction between the low field region, where $J_{\mathrm{c}}$ is limited by the grain boundaries, and the high field region, where depinning leads to dissipation.
\end{abstract}




\section{INTRODUCTION}

Neutron irradiation is certainly not practical for improving the properties of long length commercial superconductors, but a very efficient tool for benchmarking and investigating flux

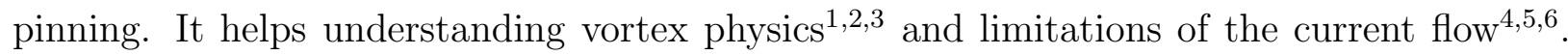
Efficient pinning centers are introduced, whose size perfectly matches the coherence length of $\mathrm{YBa}_{2} \mathrm{Cu}_{3} \mathrm{O}_{7-\delta}$ (YBCO). They improve the critical currents, if the density of defects was originally too small, but diminish them, if the total defect concentration becomes too high. Although the resulting defect structure is not perfectly well defined, since only the density of the largest defects, the collision cascades, is known ${ }^{7}$ and many smaller defects of unknown density are produced ${ }^{8}$, it is clearly established that all defects are randomly distributed and uncorrelated, i.e., without a preferred orientation. It is particularly interesting to study the interplay of these defects with the correlated disorder usually present in coated conductors or thin films 9 10,11 . In the present study, the effect of neutron irratiation on commercial coated conductors was investigated. Similarities and differences in differently produced tapes will be presented in the following in order to provide a comprehensive picture of neutron irradiation of this class of materials. We do not aim at comparing the properties of the unirradiated samples, since they were supplied at different times and the rapid development during the past few years would not allow a useful comparison between the different production routes. The different stages of optimisation turned out to be the main source of differences in the radiation response of the investigated tapes.

\section{EXPERIMENTAL}

Three different types of commercial YBCO tapes were studied. The first type of samples (denoted as MODRAB in the following) was prepared by metal organic deposition (MOD) on a RABITS template. The second series of samples (PLDYSZ) is based on an IBAD (ion beam assisted deposition) YSZ (yttrium stabilized zirconia) template. The superconducting layer was prepared by pulsed laser deposition (PLD). The third series (MOCVDMgO) had IBAD MgO templates with the YBCO layer made by metal organic chemical vapor depo-

sition (MOCVD). The MODRAB samples were irradiated to higher fluences $\left(4 \times 10^{21} \mathrm{~m}^{-2}\right.$ and $\left.10^{22} \mathrm{~m}^{-2}\right)$ than the PLDYSZ and MOCVDMgO samples $\left(2 \times 10^{21} \mathrm{~m}^{-2}\right)$. All samples 
were characterized by four probe transport prior to and after irradiation by fast neutrons. The transport measurements were made under helium gas flow up to fields of $15 \mathrm{~T}$. The samples were measured in different fields and temperatures and also rotated in the magnetic field under the maximum Lorentz force configuration $(J \perp H)$. The current was continuously ramped until an abort criterion was reached. The critical current was defined by the $1 \mu \mathrm{Vcm}^{-1}$ criterion. Indium pressed contacts are most suitable for our experiments because of their low resistivity, reliability and removability, which is important for the irradiation process. The transition temperature $\left(T_{\mathrm{c}}\right)$ and the irreversibility lines were measured with a constant current of $10 \mathrm{~mA}$, while the temperature was slowly decreased at fixed applied fields. $T_{\mathrm{c}}$ and $T_{\text {irr }}$ were evaluated at $0.1 \mu \mathrm{Vcm}^{-1}$. Only the data presented in Figs. 3 and 4 were measured in a vibrating sample magnetometer (VSM) at $10 \mathrm{~K}$, while ramping the field with $0.6 \mathrm{~T} \mathrm{~min}^{-1}$. All samples were checked by magnetoscanning $\frac{12}{2}$ to ensure homogeneous properties.

The samples were irradiated in the central irradiation facility of the TRIGA MARK II reactor in Vienna at a power of $250 \mathrm{~kW}$ (thermal/fast neutron flux density: 6/7.6 × $\left.10^{16} \mathrm{~m}^{-2} \mathrm{~s}^{-1}\right)$. The samples were sealed into a quartz tube to avoid contact between the samples and the cooling water in the reactor. The quartz tubes were put into aluminium containers, which were filled with water and placed at the correct irradiation position. The temperature during irradiation did not exceed $60{ }^{\circ} \mathrm{C}$. All fluences refer to fast neutrons $(E>$ $0.1 \mathrm{MeV})$, the corresponding thermal neutron fluences $(E<0.55 \mathrm{eV})$ were smaller by a factor of $1.27^{13}$. Neutron irradiation creates a wide spectrum of defects in the superconductor by elastic collisions of the neutrons with the atoms. Depending on the kinetic energy transferred from the neutron to the primary recoil atom, point defects or larger defect structures appear. In an avalanche like process a huge number of atoms can be displaced, leading to local melting of the crystal lattice. The result is a spherical region of amorphous material with a mean diameter of about 3 to $6 \mathrm{~nm}^{7}$. While fast neutrons $(E>0.1 \mathrm{MeV})$ transfer sufficient energy to the primary recoil atom to produce collision cascades, thermal neutrons $(E<0.55 \mathrm{eV})$ do not lead to defects in the material because their energy is below the displacement threshold. Epithermal neutrons (in the keV range) lead to the formation of point defects and point defect clusters. The density of collision cascades after irradiation to $10^{22} \mathrm{~m}^{-2}$ is about ${ }^{7}$ $5 \times 10^{22} \mathrm{~m}^{-3}$, which corresponds to a mean distance between two cascades of $27 \mathrm{~nm}$, the lattice parameter of the flux line lattice at $3.2 \mathrm{~T}$. 


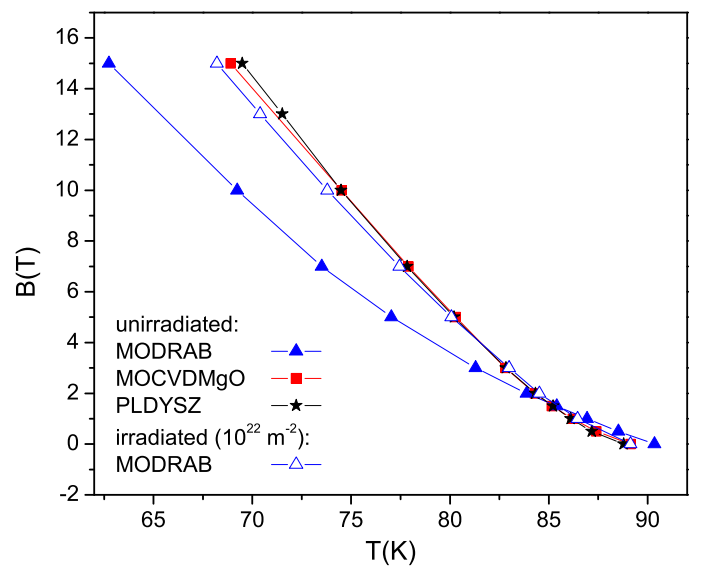

FIG. 1: The irreversibility fields $(H \| c)$ of the PLDYSZ and MOCVDMgO samples are nearly identical and higher than those of the MODRAB sample. All irreversibility lines become similar after neutron irradiaton, since only that of the MODRAB sample is changed significantly.

\section{RESULTS AND DISCUSSION}

\section{A. Transition Temperature}

The transition temperature of the MODRAB samples was $90.6 \mathrm{~K}$, of the PLDYSZ $88.5 \mathrm{~K}$ and of the MOCVDMgO 89.1 K. Neutron irradiation decreases the transition temperature, $T_{\mathrm{c}}$, which is ascribed to enhanced impurity scattering caused by the introduced defects. Nonmagnetic impurities do not reduce $T_{\mathrm{c}}$ in isotropic s-wave superconductors, but in anisotropic and d-wave superconductors $\stackrel{14}{15}$. The effect is comparatively small at the chosen fluences. We find a linear decrease with neutron fluence by around $2 \times 10^{-22} \mathrm{Km}^{2}$ (2 $\mathrm{K}$ at a fluence of $10^{22} \mathrm{~m}^{-2}$ ), which is around half that found in single crystals 2,16 . The transitions normally do not broaden after irradiation to these neutron fluences.

\section{B. Irreversibility line}

The irreversibility lines $(H \| c)$ of the samples are compared in Fig. 1. The PLDYSZ and MOCVDMgO samples behave almost identically, the irreversibility fields of the MODRAB samples are lower (except near $T_{\mathrm{c}}$ ), but shift close to the values of the other samples after 

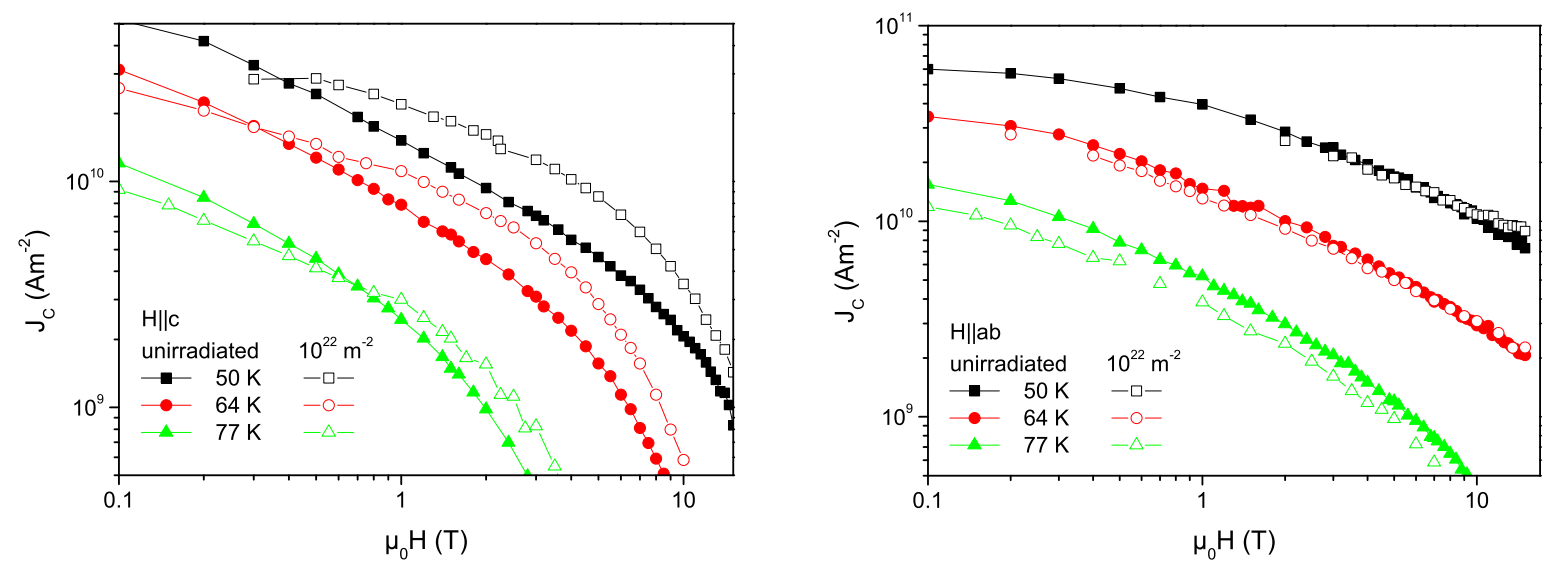

FIG. 2: Neutron irradiation enhances the critical current densities in a large field and temperature range, but decreases them at low fields or close to $T_{\mathrm{c}}$. The positive effect is larger if the field is oriented perpendicular to the tape (left panel) than in the parallel orientation (right panel).

irradiation to $10^{22} \mathrm{~m}^{-2}$. The latter do not change significantly in the accessible field range, since the moderate decrease in $T_{\mathrm{c}}$, which somewhat decreases $B_{\text {irr }}$ at high temperatures, is counterbalanced by a slight steepening of the irreversibility line. We note that neutron irradiation cannot improve the irreversibility line of state-of-the-art coated conductors and that all samples behave similarly after irradiation. It seems that the MODRAB samples have a comparatively low density of pinning efficient defects, which is increased by the irradiation, thus enhancing $B_{\text {irr }}$. The irreversibility line becomes quite insensitive to the defect concentration at high defect densities, which could indicate an upper limit for $B_{\mathrm{irr}} \underline{17}$.

The irradiation has no beneficial effect on the irreversibility line for the other main field orientation $(H \| a b)$ and we observe no significant effect (MODRAB) or a slight degradation (other samples). The variation of the intrinsic pinning potential is on the length scale of the c-axis lattice parameter (equivalent to a very high density of pinning centers) and presumably much more efficient than the cascades due to its correlated two dimensional character.

\section{Critical Currents}

Depending on field, temperature, fluence, and the original defect structure, the irradiation will enhance or decrease the critical currents. The strongest increase was observed in the 
sample with the weakest as grown pinning structure (MODRAB). The corresponding data at $77 \mathrm{~K}, 64 \mathrm{~K}$, and $50 \mathrm{~K}$ are presented in Fig. 2 for both main field orientations. The critical currents decrease at low magnetic fields after irradiation, which is discussed in detail in the next section. $J_{\mathrm{c}}(B)$ becomes flatter after the irradiation at low and intermediate fields, which leads to a crossover and an enhancement at intermediate and high fields. The flattening manifests itself in a smaller exponent $\alpha$ of the power-law $J_{\mathrm{c}} \propto B^{-\alpha}$, which is a reasonable approximation for the field dependence of $J_{\mathrm{c}}$ at intermediate fields. Note that the crossover field between the enhancement and degradation of $J_{\mathrm{c}}$ is generally higher for the field parallel to the ab planes (right panel in Fig. 2), where an enhancement is found only at high fields and low temperatures. The currents degrade in the whole field range at $77 \mathrm{~K}$. The (relative) enhancement is more pronounced at low temperatures for both orientations, which is in contrast to earlier findings on single crystals $\underline{8} \cdot \underline{16}$. This is a consequence of the competition between pinning and thermal energy, which reduces the efficiency of comparatively small defects at high temperatures. The collision cascades are obviously the largest pinning centers in single crystals, but this does not seem to be the case in coated conductors, where a large number and variety of linear and planar defects exists ${ }^{\frac{9}{}}$, which are more efficient than the cascades at high temperatures. In addition, neutron irradiation also introduces smaller defects, which are efficient only at low temperatures.

The other types of samples (PLDYSZ, MOCVDMgO) behave qualitatively similarly but data are available only for a fluence of $2 \times 10^{21} \mathrm{~m}^{-2}$ so far. A similar reduction of $J_{\mathrm{c}}$ at low fields turns to an enhancement at comparable crossover fields, which hardly depend on the neutron fluence (see Fig. 3). The field dependence becomes again weaker at intermediate field, which reduces $\alpha$. Only at high fields, the hardly changed irreversibility field causes a difference to the MODRAB samples. While the relative enhancement diverges for $H \| c$ at the originally lower irreversibility fields in the MODRAB samples, the $J_{\mathrm{c}}(B)$ curves merge again near the hardly changed irreversibility fields in the PLDSZ and MOCVDMgO samples. At high temperatures, where the irreversibility field is slightly reduced due to the decrease of $T_{\mathrm{c}}$, even a second crossover is observed, and $J_{\mathrm{c}}$ is only enhanced at intermediate fields. 


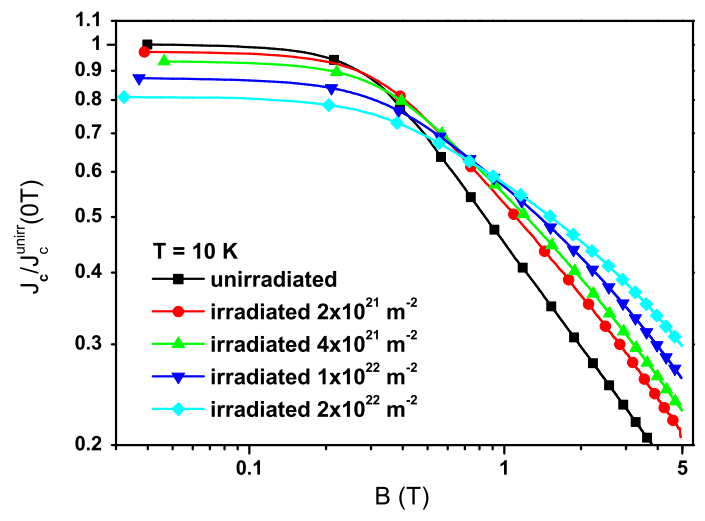

FIG. 3: Crossover in $J_{\mathrm{c}}(B)$ between the irradiated and the unirradiated state after sequential irradiation (sample MODRAB).

\section{Granularity effects}

The crossover in the field dependence $J_{\mathrm{c}}(B)$ between the unirradiated state and the irradiated state (Fig. 3), is a feature that is generally observed on conductors grown by physical as well as chemical deposition. The twofold effect of the irradiation on the current transport indicates that two regimes must be separated: a high field region $(>1 \mathrm{~T})$ which benefits from the additional pinning centres and a low field domain, where a different mechanism causes a $J_{\mathrm{c}}$-depression. This is explained by considering the specific nature of current transport in a granular medium, i.e., the critical current density in the grains $J_{\mathrm{c}, \mathrm{G}}$ has to be distinguished from the grain boundary critical current density $J_{\mathrm{c}, \mathrm{GB}}$ flowing across the sample. Numerous experiments on bicrystals $\frac{18}{18}$ showed that, depending on the grain boundary misorientation angle, the current transport is controlled by $J_{c, \text { GB }}$ at low fields, whereas at fields in the Tesla range $J_{\mathrm{c}, \mathrm{G}}$ limits the current transport. Although it is not fully established that the behaviour of a single well-defined grain boundary is representative for the complex grain boundary network of a coated conductor ${ }^{19}, 20$, the current limitation by the grains and an increase of $J_{\mathrm{c}, \mathrm{G}}$ after irradiation are straightforward explanations for the observed $J_{\mathrm{c}}$ improvement in high fields. Further evidence for this scenario is provided by neutron irradiation of YBCO thin films grown on single-crystalline substrates, which can be regarded as single macroscopic grains and thus serve as a model system for the individual microscopic grains in a coated conductor. These films show, in agreement with the above 
assumption of an increase in $J_{\mathrm{c}, \mathrm{G}}$, enhanced critical current densities in the entire field range (cf. Fig. 4a and reference 21), if the temperature is not too close to the superconducting transition temperature, i.e., when the (moderate) reduction of $T_{\mathrm{c}}$ after irradiation is negligible. The fact that the $J_{\mathrm{c}}$ decrease occurs at rather low fields, where $J_{\mathrm{c}, \mathrm{GB}}$ is dominant in the bicrystal experiments, suggests that the grain boundary network is responsible for this effect. Two different but not mutually exclusive mechanisms are able to explain the reduction of $J_{\mathrm{c}, \mathrm{GB}}$. First, it is important to notice, that the density of the irradiation defects is too low to reduce the cross-section for current transport significantly. But the defects are mobile during the irradiation and the grain boundaries represent natural barriers for their movement, which causes the defects to accumulate - the grain boundaries deteriorate after irradiation. This effect has, however, only an impact on the overall $J_{\text {c }}$, if the current transport is limited by the grain boundaries. Hence, the crossover indicates the transition between the low field regime, which is dominated by the grain boundary network, and the high-field regime controlled by the properties of the grains.

Another explanation for the $J_{\mathrm{c}}$ reduction at low fields is based on the field exerted by the intra-granular shielding currents on the grain boundaries: If $J_{\mathrm{c}, \mathrm{G}}$ is larger than $J_{\mathrm{c}, \mathrm{GB}}$, additional flux remains trapped in the grains after a change of the external applied field and the circulating currents in the grain generate an additional field at the grain boundaries. Thus, the enhancement of $J_{\mathrm{c}, \mathrm{G}}$ by the irradiation, which is responsible for the $J_{\mathrm{c}}$ improvement at high fields, increases the grain boundary field and thereby reduces the field dependent $J_{\mathrm{c}, \mathrm{GB}}$ at low applied fields. An experimental test is provided by a method similar to Ref. 22, where the remnant magnetic moment $m_{\text {rem }}$ of the sample is assessed after applying successively larger fields $H_{\max }$. If $J_{\mathrm{c}, \mathrm{GB}}$ is smaller than $J_{\mathrm{c}, \mathrm{G}}$, flux will initially penetrate along the grain boundaries. After the grain boundary network has saturated and additional flux is trapped only in the grains, $m_{\mathrm{rem}}\left(H_{\max }\right)$ decreases as the intra-grain currents increase the field at the grain boundary. ${ }^{26}$ The expected behaviour of $m_{\text {rem }}\left(H_{\max }\right)$ is clearly evident in samples grown at an early stage of optimisation (Fig. 4b) to the granular substrate and thus carry a rather low $J_{\mathrm{c}, \mathrm{GB}}{ }^{21}$. Despite the smaller $J_{\mathrm{c}}$ of this sample, the crossover occurs at fields comparable to state-of-the-art coated conductors. Although the two proposed mechanisms are different, they do not exclude each other, but rely on the same argument: a transition between grain boundary to grain controlled current transport leading to a crossover in $J_{\mathrm{c}}(B)$ between the irradiated and the unirradiated state. It should further be noted that the complexity of the 
(a)

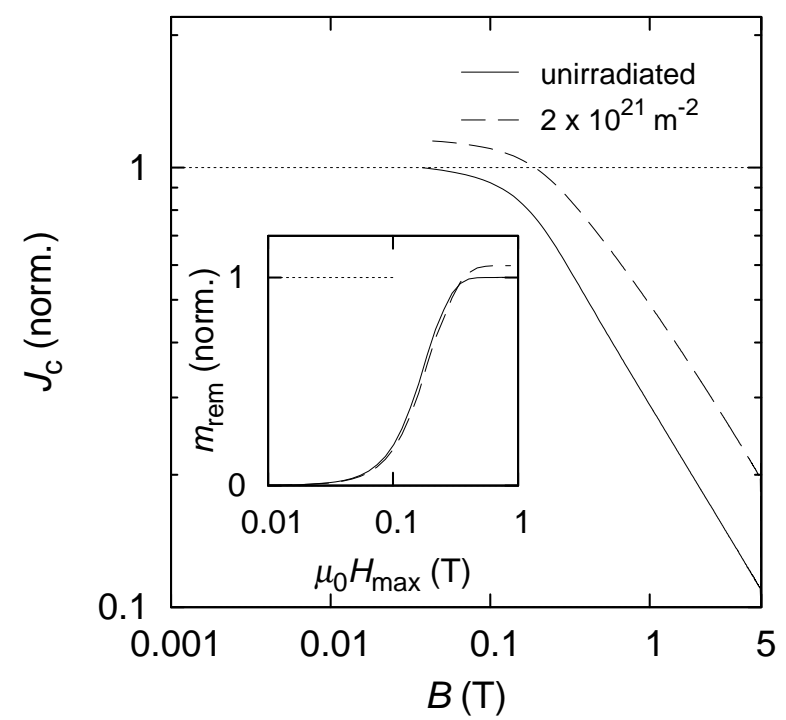

(b)

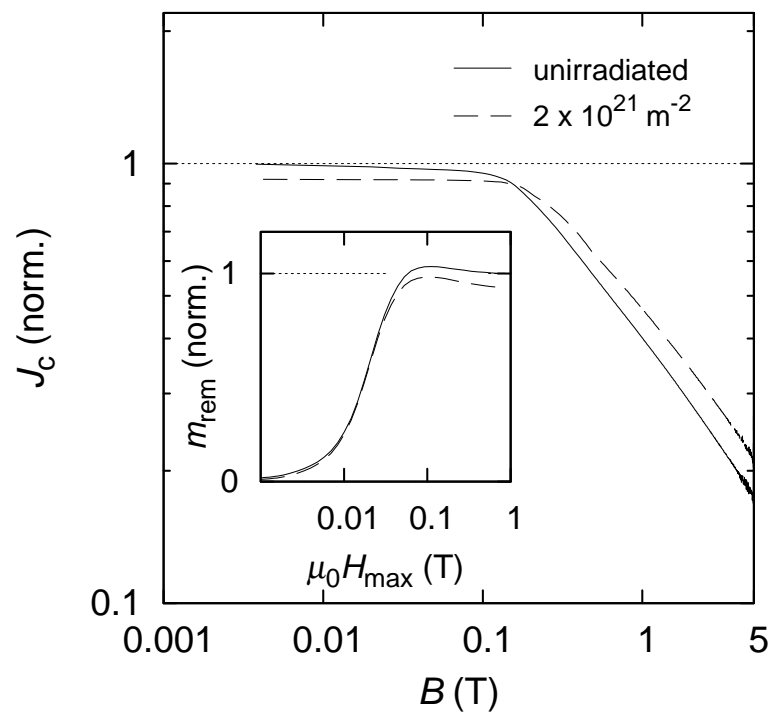

FIG. 4: Comparison of the effect of neutron irradiation on samples grown on single-crystalline (a) and poly-crystalline (b) substrates. The critical current density in the single-crystalline sample is enhanced in the entire field range, while the granular sample exhibits a crossover at about the same field as state-of-the-art coated conductors. The inset shows that the remnant moment of the granular sample decreases when the grain boundary currents are saturated and the flux penetrates the grains. (Data are normalised to the unirradiated state.)

grain boundary network does not allow to draw clear distinctions and the crossover indicates only approximately the transition from one regime to the other.

\section{E. Angular dependence of $J_{\mathrm{c}}$}

The angular dependence of the critical current in the MODRAB sample at $64 \mathrm{~K}$ and $3 \mathrm{~T}$ is presented in Fig. 5. The pronounced peak at $0^{\circ}$ either comes from intrinsic pinning by the periodic variation of the order parameter along the c-axis, or from correlated defects, such as stacking faults. There is hardly any trace of a peak at $90^{\circ}$ in the unirradiated tape. This indicates that the density of c-axis correlated defects is small. The random, uncorrelated disorder introduced by the neutrons reduces the $I_{\mathrm{c}}$-anisotropy, $\gamma_{I_{\mathrm{c}}}=I_{\mathrm{c}}^{\max } / I_{\mathrm{c}}^{\min }$, from 2.7 to 1.3 at a fluence of $10^{22} \mathrm{~m}^{-2}$. The latter is considerably smaller than the $I_{\mathrm{c}}$-anisotropy in a thin film with a claimed (intrinsic or effective) anisotropy of only $\underline{10} 1.5$, obtained by a scaling 


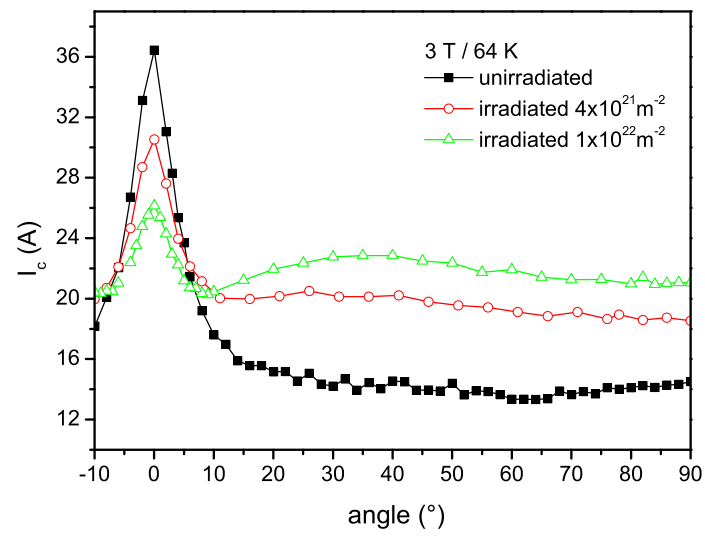

FIG. 5: The irradiation reduces the $J_{\mathrm{c}^{-}}$anisotropy (sample MODRAB). The minimum in $J_{\mathrm{c}}$ appears near $9^{\circ}$, where the currents are hardly changed by the introduced defects.

analysis $J_{\mathrm{c}}(\theta)^{\underline{10}}$ on the basis of the ansiotropic scaling approach by Blatter et al. ${ }^{23}$ Such a small intrinsic anisotropy can be excluded in the present sample, since the anisotropy of the irreversibility fields is much higher and not significantly altered by the irradiation. The shape of $J_{\mathrm{c}}(\theta)$ in the irradiated state is extremely interesting. The peak at $0^{\circ}$ is followed by a minimum at about $9^{\circ}$ and another broad maximum centered at around $\sim 35^{\circ}$. Note that this behaviour was induced by the addition of random, uncorrelated disorder and is hardly compatible with the anisotropic scaling approach, although this model assumes such a defect structure, but with weak instead of strong pinning defects. This is not unexpected, since the scaling of $J_{\mathrm{c}}$ is based on single-vortex weak-collective pinning ${ }^{23}$ which predicts extremely small currents and is obviously not applicable to "high- $J_{\mathrm{c}}$ " coated conductors.

The MOCVDMgO samples contain c-axis correlated defects, as indicated by the peak at around $90^{\circ}$ shown in Fig. 6. This peak disappears after irradiation and the currents hardly change between $25^{\circ}$ and $90^{\circ}$ at $64 \mathrm{~K}$ and $4 \mathrm{~T}$, in contradiction to the anisotropic scaling approach. The minimum, again at about $9^{\circ}$, which was present in these samples also before irradition, is amplified. Only the intrinsic pinning remains practically unaffected.

At higher fields (not shown in Fig. 6) , a minimum occurs at $90^{\circ}$, which is an obvious consequence of the small irreversibility field in this orientation. The ratio of the irreversiblity fields in the main field orientations is consistent with anisotropic scaling and with expectations for the intrinsic anisotropy $\left(B_{\mathrm{irr}}^{a b} / B_{\mathrm{irr}}^{c} \sim 4\right)$ and seems to represent a much more reliable 


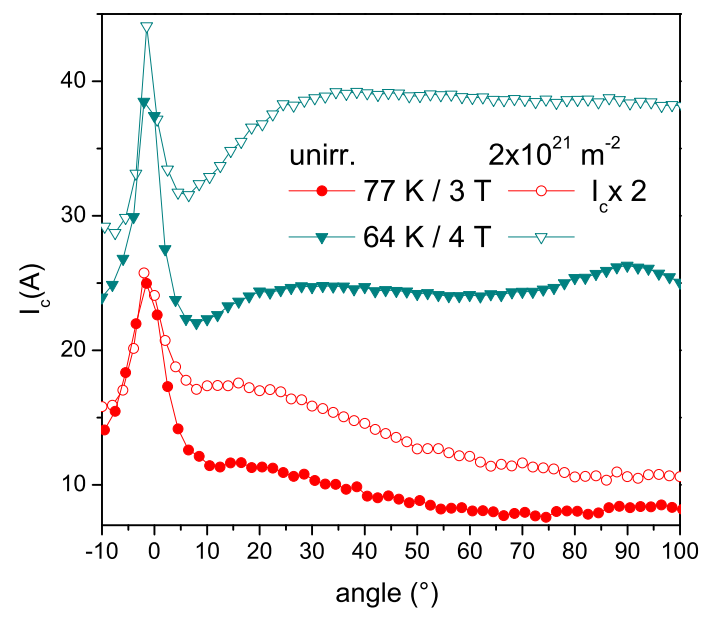

FIG. 6: The peak at $90^{\circ}$ indicates the presence of c-axis correlated defects in the MOCVDMgO sample. It disappears after neutron irradiation, the minimum at $\sim 9^{\circ}$ is amplified.

estimate for the intrinsic anisotropy (the scaling of fields is independent of pinning models) than the angular dependence of $J_{\mathrm{c}}$, since this approach does not work for the relevant pinning centers in coated conductors.

\section{F. Coated conductors for fusion magnets}

Powerful magnets will be needed to confine the plasma of future fusion power plants. The use of high temperature superconductors instead of $\mathrm{Nb}_{3} \mathrm{Sn}$ would allow operation at higher temperatures and/or fields, which would reduce the cooling requirements and could also result in a more compact and cheaper design. However, only first concepts for fusion magnets made of coated conductors are being developed at present. Cables with a current capability in the ten $\mathrm{kA}$ range are needed and the strands have to be somehow twisted ${ }^{24}$ in order to reduce ac losses. Electrical, mechanical, and thermal stabilization of such wires and quench detection/protection will be demanding issues. Apart from these technological issues, the superconducting properties have to fulfill two basic requirements. The critical current densities must be comparatively high, since the superconducting volume fraction of a coated conductor is low (typically 1-2\%) and they must not degrade during operation in a hard radiation environment (fast neutrons). A rough estimate of the required intrinsic critical 


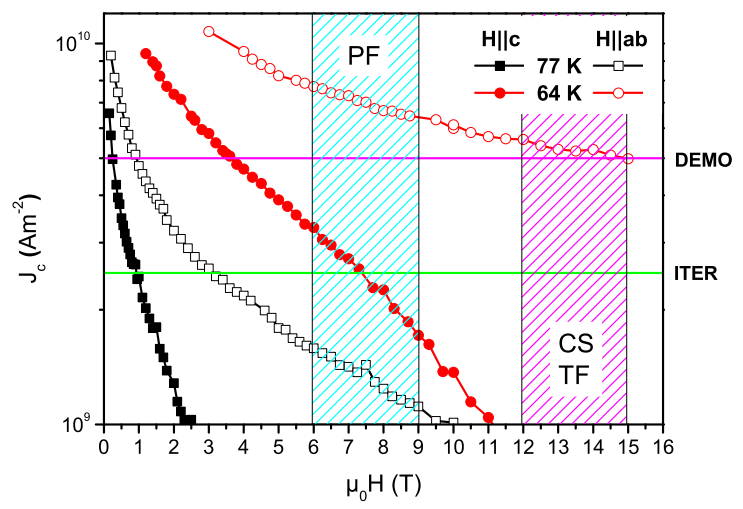

FIG. 7: Commercial state-of-the-art conductors fulfill the requirements of fusion magnets on $J_{\mathrm{c}}$ at $64 \mathrm{~K}$, a temperature, where liquid nitrogen (at low pressure) can be used as coolant, if the magnetic field is parallel to the ab planes. For the other main field orientation as well as at $77 \mathrm{~K}$ the performance is still too low.

current density leads to about $2.5 \times 10^{9} \mathrm{Am}^{-2}\left(5 \times 10^{9} \mathrm{Am}^{-2}\right)$ at about $12 \mathrm{~T}(14 \mathrm{~T})^{25}$, assuming the overall current density of the actual ITER (DEMO) cable design for the toroidal field (TF) coils or the central solenoid (CS). These values are certainly achievable with present conductors, but define the operation temperature. Cooling with liquid nitrogen would be favorable, but is possible only above $64 \mathrm{~K}$ (at low pressure). Figure 7 demonstrates that this seems feasible (although not at $77 \mathrm{~K}$ ) with present conductors (MOCVDMgO) if the field is aligned with the ab planes. The magnetic field in the fusion coils will be close to this orientation, but, depending on the cable and coil design, orthogonal components will occur. In the other limiting case $(H \| c)$, the performance currently fails significantly. Since the currents drop quickly at small misalignment angles (Figs. 5] and 6), further conductor development seems mandatory in order to reach the required current densities at these high temperatures. The field in the poloidal field $(\mathrm{PF})$ coils is much smaller (up to about $6 \mathrm{~T}$ in ITER and $9 \mathrm{~T}$ in DEMO), which is also highlighted in Fig. 7. Even these requirements seem to be demanding at $77 \mathrm{~K}$ or for $H \| c$. The expected life time fluence (fast neutrons) at the main fusion magnets (PF, CS) is around $2 \times 10^{22} \mathrm{~m}^{-2}$ and might be even higher in future fusion power plants. Since the currents at $77 \mathrm{~K}$ already degrade at a fluence of $10^{22} \mathrm{~m}^{-2}$, radiation resistance is an important issue for the application of coated conductors in fusion 
magnets, at least at elevated temperatures.

\section{CONCLUSIONS}

The influence of fast neutron irradiation on differently fabricated coated conductors was found to be similar. The critical currents decrease at low magnetic fields, where intergranular currents set the upper limit for loss free current flow. This decrease can be understood either by a slight degradation of the grain boundaries, or by the increasing shielding currents within the grains, which generate an additional field at the grain boundaries.

The irradiation enhances $J_{\mathrm{c}}$ at intermediate and high magnetic fields, where the macroscopic current is limited by pinning, which is improved by the additional pinning centers. Although these pinning centers are uncorrelated, the angular dependence of $J_{\mathrm{c}}$ does not obey anisotropic scaling. This is a direct consequence of the pinning strength of these defects, which cannot be considered as weak, as assumed in the scaling approach. Since this should be true for any relevant pinning centers in these "high- $J_{\mathrm{c}}$ " conductors, anisotropic scaling based on weak collective single vortex pinning is not applicable.

The irreversibility line in the best available YBCO coated conductors seems to be close to optimum, because a higher concentration of strong defects does not lead to further improvements.

\section{Acknowledgments}

This work, supported by the European Communities under the contract of Association between EURATOM/OEAW was partly carried out within the framework of the European Fusion Development Agreement. The views and opinions expressed herein do not necessarily reflect those of the European Commission. One of us (M.C.) wishes to acknowledge financial support through the NESPA project.

* Electronic address: eisterer@ati.ac.at

1 Meier-Hirmer R, Küpfer H, and Scheurer H 1985 Phys. Rev. B 31183

2 Werner M, Sauerzopf F M, Weber H W, and Wisniewski A 2000 Phys. Rev. B 6114795 
3 Zehetmayer M, Eisterer M, Jun J, Kazakov S M, Karpinski J, Birajdar B, Eibl O, and Weber H W 2004 Phys. Rev. B 69054510

4 Tönies S, Vostner A, and Weber H W 2002 J. Appl. Phys. 922628

5 Eisterer M, Haindl S, Zehetmayer M, Gonzalez-Arrabal R, Weber H W, Litzkendorf D, Zeisberger M, Habisreuther T, Gawalek W, Shlyk L, and Krabbes G 2006 Supercond. Sci. Technol. $19 \mathrm{~S} 530$

6 Eisterer M, Müller R, Schöppl R, Weber H W, Soltanian S, and Dou S X 2007 Supercond. Sci. Technol. 20117

7 Frischherz M C, Kirk M A, Zhang J P, and Weber H W 1993 Philos. Mag. A 671347

8 Sauerzopf F M 1998 Phys. Rev. B 5710959

9 Foltyn S R, Civale L, MacManus-Driscoll J L, Jia Q X, Maiorov B, Wang H, and Maley M 2007 Nat. Mat. 6631

10 Gutierrez J, Llordes A, Gazquez J, Gibert M, Roma N, Ricart S, Pomar A, Sandiumenge F, Mestres N, Puig T, and Obradors X 2007 Nat. Mat. 6367

11 Chen Z, Kametani F, Chen Y, Xie Y, Selvamanickam V, and Larbalestier D C 2009 Supercond. Sci. Technol. 22055013

12 Fuger R, Hengstberger F, Eisterer M, and Weber H W 2007 IEEE Trans. Appl. Supercond. 17 3753

13 Weber H W, Böck H, Unfried E, and Greenwood L R 1986 J. Nucl.Mater. 137236

14 Millis A J, Sachdev S, and Varma C M 1988 Phys. Rev. B 374975

15 Radtke R J, Levin K, Schüttler H-B, and Norman M R 1993 Phys. Rev. B 48653

16 Sauerzopf F M, Wiesinger H P, Kritscha W, Weber H W, Crabtree G W, and Liu J Z 1991 Phys. Rev. B 433091

17 Figueras J, Puig T, Obradors X, Kwok W K, Paulius L, Crabtree G W, and Deutscher G 2006 Nat. Phys. 2402

18 Hilgenkamp H and Mannhart J 2002 Rev. Mod. Phys. 74485

19 Feldmann D M, Holesinger T G, Feenstra R, and Larbalestier D C 2008 J. Am. Ceram. Soc. 911869

20 Durrell J H and Rutter N A 2009 Supercond. Sci. Technol. 22013001

21 Hengstberger F, Eisterer M, Weber H W, Kursumovic A, and MacManus-Driscoll J L 2007 IEEE Trans. Appl. Supercond. 173549 
22 Müller K-H, Andrikidis C, Liu H K, and Dou S X 1994 Phys. Rev. B 5010218

23 Blatter G, Geshkenbein V B, and Larkin A I 1992 Phys. Rev. Lett. 68875

24 Goldacker W, Frank A, Kudymow A, Heller R, Kling A, and Terzieva S, and Schmidt C 2009 Supercond. Sci. Technol. 22034003

25 Fuger R, Eisterer M, and Weber H W 2009 IEEE Trans. Appl. Supercond. 191532

26 Note, that the contribution of the currents in the grains to $m_{\mathrm{rem}}$ is not significant, because $m \propto r^{3}$, where $r$ is the radius, which is a few millimetres for the sample and more than a hundred times less for a single grain. Thus, the dominating component of the total magnetic moment stems from the currents flowing across the grain boundaries in all samples expect those with high-angle grain boundaries, which makes a direct assessment of $J_{\mathrm{c}, \mathrm{G}}$ and $J_{\mathrm{c}, \mathrm{GB}}$ (as for example in Ref. 4) impossible. 